\title{
HANKEL MEASURES ON HARDY SPACE
}

\section{JIE XIAO}

We characterise the complex measures $\mu$ on the open unit disk $\mathbf{D}$ such that $\left|\int_{\mathrm{D}} f^{2} d \mu\right| \leqslant C\|f\|_{H^{2}}^{2}$ for all $f$ in the Hardy space $H^{2}$. The characterisation involves Carleson measures, the duality between $H^{1}$ and $B M O A$, and Hankel operators.

Let $\mathbf{D}$ be the unit disk $\{z:|z|<1\}$ in the complex plane $\mathbf{C}$ and denote by $d m$ the two-dimensional Lebesgue measure on $\mathbf{D}$. The boundary $\{z:|z|=1\}$ of $\mathbf{D}$ will be written as $\partial \mathbf{D}$.

For $p \in[1, \infty)$, define $H^{p}$ to be the Hardy space of all holomorphic functions $f$ on $\mathbf{D}$ for which

$$
\|f\|_{H^{p}}=\sup _{r \in(0,1)}\left[\frac{1}{2 \pi} \int_{\partial \mathbf{D}}|f(r \zeta)|^{p}|d \zeta|\right]^{1 / p}<\infty .
$$

If $f \in H^{p}$, the radial limit $\lim _{r \rightarrow 1} f(r \zeta)$, written $f(\zeta)$, exists for almost every $\zeta \in \partial \mathbf{D}$; we may thus identify $f$ with its boundary value.

The Fefferman-Stein duality theorem [4] tells us that $\left[H^{1}\right]^{*} \cong B M O A$ under the pairing:

$$
\langle f, g\rangle=\frac{1}{2 \pi} \int_{\partial \mathbf{D}} f(\zeta) \overline{g(\zeta)}|d \zeta|,
$$

where $B M O A$ is the space of all $f \in H^{1}$ such that

$$
\|f\|_{B M O A}=|f(0)|+\sup _{w \in \mathbf{D}}\left\|f \circ \phi_{w}-f(w)\right\|_{H^{1}}<\infty,
$$

and $\phi_{w}: \mathbf{D} \rightarrow \mathbf{D}$ is the Möbius transformation $z \mapsto(w-z) /(1-\bar{w} z)$, which interchanges $w$ and 0 (see [1]).

When the author visited Lund University, Sweden, J. Peetre posed the following problem:

\section{Received 18th October, 1999}

The author would like to take this opportunity to thank J. Peetre, K.J. Wirths and G.K. Zhang for their illuminating suggestions. The author is grateful to the referee and $\mathrm{M}$. Cowling for several helpful comments on previous versions of the article. In addition, thanks are given to the AvH-foundation of Germany and the NNSF (No. 19771006) of China for their support.

Copyright Clearance Centre, Inc. Serial-fee code: 0004-9727/00 \$A2.00+0.00. 
Peetre's Problem. Let $\mu$ be a complex-valued measure on D. What geometric properties must $\mu$ have in order that

$$
\left|\int_{\mathrm{D}} f^{2} d \mu\right| \leqslant C\|f\|_{H^{2}}^{2} \quad \forall f \in H^{2} \quad ?
$$

Here and throughout the article, the letter $C$ stands for a (variable) positive constant.

Before providing an answer to the problem, we would like to make two observations. First, if $I$ is a subarc of $\partial \mathrm{D}$ with arclength $|I|$, then $S(I)$ denotes the Carleson box

$$
\left\{r \zeta \in \mathbf{D}: 1-\frac{|I|}{2 \pi} \leqslant r<1, \zeta \in I\right\} \text {. }
$$

A complex measure $\mu$ on $\mathrm{D}$ satisfying

$$
\sup _{I \subset \partial \mathbf{D}} \frac{|\mu|(S(I))}{|I|}<\infty
$$

is called a Carleson measure (see [7]). It is known that $\mu$ is a Carleson measure (see $[5$, p.63], $[10$, p.170]) if and only if

$$
\int_{\mathrm{D}}|f|^{2} d|\mu| \leqslant C\|f\|_{H^{2}}^{2} \quad \forall f \in H^{2}
$$

A complex measure $\mu$ on D that satisfies condition (1) will be called a Hankel measure, because Peetre's problem originates from the study of Hankel matrices (see also [6, 9]). It is clear that a Carleson measure must be a Hankel measure, but not conversely.

Second, any Hankel measure is Möbius invariant in the following sense. Let $\phi$ : $\mathbf{D} \rightarrow \mathbf{D}$ be the Möbius mapping $z \mapsto(a z+b) /(\bar{b} z+\bar{a})$, where $a, b \in \mathbf{C}$ and $|a|^{2}-|b|^{2}=$ 1. Then if $f \in H^{2}$, so too is the function $\tilde{f}: z \mapsto[f \circ \phi(z)] /(\bar{b} z+\bar{a})$, and the mapping $f \mapsto \tilde{f}$ is an isometry on $H^{2}$. So if $d \mu(z)$ changes to $(d \mu \circ \phi(z))(\bar{b} z+\bar{a})^{2}$, then the best constant in Peetre's problem does not change. This feature corresponds to the conformal invariance of Carleson measures. See for example [5, p.239].

Before we state our solution to Peetre's problem, we need two more definitions. Given a measure $\mu$ on $\mathbf{D}$, we define the function $P_{\mu}$ and the Hankel operator $K_{\mu}$ (see [8]) by the formulas

$$
\begin{aligned}
P_{\mu}(z) & =\int_{\mathrm{D}} \frac{1}{1-z \bar{w}} d \mu(w) \\
\left(K_{\mu} f\right)(z) & =\int_{\mathrm{D}} \frac{f(w)}{1-w z} d \mu(w) .
\end{aligned}
$$


THEOREM. Let $\mu$ be a complex measure on $\mathrm{D}$. Then the following are equivalent:

(a) $\mu$ is a Hankel measure.

(b) $\left|\int_{\mathrm{D}} f d \mu\right| \leqslant C\|f\|_{H^{1}} \quad \forall f \in H^{1}$.

(c) $P_{\bar{\mu}}$ is in $B M O A$.

(d) $\sup _{I \subset \partial \mathrm{D}} \frac{1}{|I|} \int_{S(I)}\left|\int_{\mathrm{D}} \frac{\bar{w} d \bar{\mu}(w)}{(1-\bar{w} z)^{2}}\right|^{2}\left(1-|z|^{2}\right) d m(z)<\infty$.

(e) $\left|\int_{\mathbf{D}} f_{1} f_{2} d \mu\right| \leqslant C\left\|f_{1}\right\|_{H^{2}}\left\|f_{2}\right\|_{H^{2}} \quad \forall f_{1}, f_{2} \in H^{2}$.

(f) $K_{\mu}$ is a bounded operator on $H^{2}$.

(g) There exists $F \in L^{\infty}(\partial \mathrm{D})$ such that

$$
\int_{\mathbf{D}} f d \mu=\frac{1}{2 \pi i} \int_{\partial \mathbf{D}} f(\zeta) F(\zeta) d \zeta \quad \forall f \in H^{\mathbf{1}} .
$$

(h) $\sup _{\boldsymbol{A}}\left|\int_{\mathbf{D}} A d \mu\right|<\infty$, where the supremum ranges over all holomorphic atoms.

Proof: Step 1. We show that (a), (b), (c) and (d) are equivalent.

Trivially, (b) implies (a). On the other hand, suppose (a) holds. To prove (b), take $f \in H^{1}$ with $f \not \equiv 0$. By [3, Theorem 2.8, p.24], there exist an inner factor $g$ and an outer factor $h$ such that $f=g h$ and $\|h\|_{H^{1}} \leqslant\|f\|_{H^{1}}$. Set $f_{1}=(g-1) h / 2$ and $f_{2}=(g+1) h / 2$. Then $f=f_{1}+f_{2}$, and $\left\|f_{k}\right\|_{H^{1}} \leqslant\|f\|_{H^{1}}, k=1,2$. Since both $f_{1}$ and $f_{2}$ are not equal to 0 anywhere on $\mathbf{D}$, there are $g_{1}, g_{2} \in H^{2}$ such that $f_{1}=g_{1}^{2}$ and $f_{2}=g_{2}^{2}$. Consequently,

$$
\left|\int_{\mathbf{D}} f d \mu\right| \leqslant\left|\int_{\mathbf{D}} g_{1}^{2} d \mu\right|+\left|\int_{\mathbf{D}} g_{2}^{2} d \mu\right| \leqslant C\left(\left\|g_{1}\right\|_{H^{2}}^{2}+\left\|g_{2}\right\|_{H^{2}}^{2}\right) \leqslant C\|f\|_{H^{1}},
$$

that is, (b) holds.

By definition of $P_{\bar{\mu}}$,

$$
\begin{aligned}
\int_{\mathbf{D}} f(z) d \mu(z) & =\int_{\mathbf{D}}\left[\frac{1}{2 \pi} \int_{\partial \mathbf{D}} \frac{f(\zeta)}{1-z \bar{\zeta}}|d \zeta|\right] d \mu(z) \\
& =\frac{1}{2 \pi} \int_{\partial \mathbf{D}} f(\zeta)\left[\int_{\mathbf{D}} \frac{1}{1-z \bar{\zeta}} d \mu(z)\right]|d \zeta| \\
& =\frac{1}{2 \pi} \int_{\partial \mathbf{D}} f(\zeta) \overline{P_{\bar{\mu}}(\zeta)}|d \zeta| \\
& =\left\langle f, P_{\bar{\mu}}\right\rangle .
\end{aligned}
$$


Thus, (c) holds if and only if (b) does, by the isomorphism between $\left[H^{1}\right]^{*}$ and $B M O A$.

An $H^{2}$-function $f$ belongs to $B M O A$ if and only if $\left(1-|z|^{2}\right)\left|f^{\prime}(z)\right|^{2} d m(z)$ is a Carleson measure (see $[10$, p.178]). Accordingly, (c) is equivalent to (d).

STEP 2. We verify that (b), (e) and (f) are equivalent.

An application of Schwarz's inequality to $f_{1} f_{2}$ (where $f_{1}, f_{2} \in H^{2}$ ) shows that (b) implies (e). On the other hand, let (e) hold. By [5, p.87, Exercise 1], we see that every $f \in H^{1}$ can be factored as $f=f_{1} f_{2}$ with $f_{1}, f_{2} \in H^{2}$ and $\left\|f_{1}\right\|_{H^{2}}^{2}=\left\|f_{2}\right\|_{H^{2}}^{2}=\|f\|_{H^{1}}$, so that (b) follows from the estimate

$$
\left|\int_{\mathbf{D}} f d \mu\right| \leqslant C\left\|f_{1}\right\|_{H^{2}}\left\|f_{2}\right\|_{H^{2}}=C\|f\|_{H^{1}} .
$$

When $f, g \in H^{2}$, the definition of $K_{\mu}$ implies that

$$
\begin{aligned}
\left\langle K_{\mu} f, g\right\rangle & =\frac{1}{2 \pi} \int_{\partial \mathbf{D}}\left(K_{\mu} f\right)(\zeta) \overline{g(\zeta)}|d \zeta| \\
& =\frac{1}{2 \pi} \int_{\partial \mathbf{D}}\left[\int_{\mathbf{D}} \frac{f(w)}{1-\zeta w} d \mu(w)\right] \overline{g(\zeta) \mid} d \zeta \mid \\
& =\int_{\mathbf{D}} f(w)\left[\frac{1}{2 \pi} \int_{\partial \mathbf{D}} \frac{\overline{g(\zeta)}}{1-\zeta w}|d \zeta|\right] d \mu(w) \\
& =\int_{\mathbf{D}} f(w) \overline{g(\bar{w})} d \mu(w) .
\end{aligned}
$$

This formula shows that (e) and (f) are equivalent.

STEP 3. We prove the equivalence of $(\mathrm{b}),(\mathrm{g})$ and $(\mathrm{h})$.

It is obvious that $(\mathrm{g})$ implies $(\mathrm{b})$, thanks to the inequality

$$
\left|\int_{\mathbf{D}} f d \mu\right|=\left|\frac{1}{2 \pi i} \int_{\partial \mathbf{D}} f(\zeta) F(\zeta) d \zeta\right| \leqslant\|F\|_{\infty}\|f\|_{H^{1}} \quad \forall f \in H^{1} .
$$

Conversely, if (b) is valid, then the linear functional $T$, defined by $T(f)=\int_{\mathbf{D}} f d \mu$, belongs to $\left[H^{1}\right]^{*}$. The Hahn-Banach theorem allows us to extend $T$ to $L^{1}(\partial \mathrm{D})$, and hence produce $G \in L^{\infty}(\partial \mathrm{D})$ such that

$$
T(f)=\frac{1}{2 \pi} \int_{\partial \mathbf{D}} f(\zeta) \overline{G(\zeta)}|d \zeta| \quad \forall f \in L^{1}(\partial \mathbf{D}) .
$$

Take $F$ such that $F(z)=\overline{z G(z)}$; then $F \in L^{\infty}(\partial \mathrm{D})$ and

$$
\int_{\mathbf{D}} f d \mu=T(f)=\frac{1}{2 \pi i} \int_{\partial \mathbf{D}} f(\zeta) F(\zeta) d \zeta \quad \forall f \in H^{1},
$$


and $(\mathrm{g})$ holds.

In [2], atoms are defined to be functions $a: \partial \mathrm{D} \rightarrow \mathbf{C}$ which are either identically 1 or are supported on an open subarc $I$ of $\partial \mathbf{D}$, and satisfy

$$
\|a\|_{\infty} \leqslant \frac{1}{|I|} \text { and } \int_{\partial \mathbf{D}} a(\zeta)|d \zeta|=0 .
$$

Holomorphic atoms $A$ are the holomorphic projection of atoms $a$, that is,

$$
A(z)=\frac{1}{2 \pi} \int_{\partial \mathrm{D}} \frac{a(\zeta)}{1-\bar{\zeta} z}|d \zeta|
$$

By [2, Theorem V], every $f \in H^{1}$ can be represented as $\sum_{j=1}^{\infty} \lambda_{j} A_{j}$, where $A_{j}$ are holomorphic atoms and $\lambda_{j} \in \mathrm{C}$ with $\sum_{j=1}^{\infty}\left|\lambda_{n}\right| \leqslant C\|f\|_{H^{1}}$. This decomposition, together with [2, Proposition VI], demonstrates that (b) implies (h) and vice versa. The proof of the theorem is complete.

REMARK. Since $f_{w}(z)=\left(1-|w|^{2}\right) /(1-\bar{w} z)^{2}$ lies in $H^{1}$ and $\sup _{w \in \mathbf{D}}\left\|f_{w}\right\|_{H^{1}}<\infty$, a necessary condition for $\mu$ to be a Hankel measure is that

$$
\sup _{w \in \mathbb{D}}\left|\int_{\mathbf{D}} \frac{1-|w|^{2}}{(1-\bar{w} z)^{2}} d \mu(z)\right|<\infty .
$$

We do not know whether this condition is sufficient, too. However, it is worth mentioning (see $[5$, p.139]) that $\mu$ is a Carleson measure if and only if

$$
\sup _{w \in D} \int_{D} \frac{1-|w|^{2}}{|1-\bar{w} z|^{2}} d|\mu|(z)<\infty
$$

As in [6] or [8], it is not hard to check that $K_{\mu}$ is a classical Hankel operator on $H^{2}$, and hence $K_{\mu}: H^{2} \rightarrow H^{2}$ is a compact or $S_{p}$ (the Schatten-von Neumann $p$-class, for $p \in(1, \infty))$ operator if and only if $P_{\bar{\mu}}$ belongs to $V M O A$, the space of all $f \in H^{1}$ with

$$
\lim _{w \rightarrow \partial D}\left\|f \circ \phi_{w}-f(w)\right\|_{H^{1}}=0
$$

or to $B_{p}$, the $p$-Besov space of all holomorphic functions $f$ on $\mathrm{D}$ such that

$$
\int_{\mathbf{D}}\left|f^{\prime}(z)\right|^{p}\left(1-|z|^{2}\right)^{p-2} d m(z)<\infty
$$

respectively. 


\section{REFERENCES}

[1] A. Baernstein II, 'Analytic functions of bounded mean oscillation', in Aspects of contemporary complex analysis (Academic Press, New York, 1980), pp. 3-36.

[2] R. Coifman, R. Rochberg and G. Weiss, 'Factorization theorems for Hardy spaces in several complex variables', Ann. Math. 103 (1976), 611-635.

[3] P.L. Duren, Theory of $H^{p}$ spaces (Academic Press, New York, 1970).

[4] C. Fefferman and E.M. Stein, ' $H^{p}$ spaces of several variables', Acta Math. 129 (1972), 137-193.

[5] J. Garnett, Bounded analytic functions, Pure and Applied Mathematics 96 (Academic Press, Inc., New York, London, 1981).

[6] S. Janson and J. Peetre, 'A new generalization of Hankel operators', Math. Nachr. 132 (1987), 313-328.

[7] P. Jones, ' $L^{\infty}$ estimates for the $\bar{\partial}$ problem in a half-plane', Acta Math. 150 (1983), 137-152.

[8] D.H. Luecking, 'Trace ideal criteria for Toeplitz operators', J. Funct. Anal. 73 (1987), 345-368.

[9] H. Widom, 'Hankel matrices', Trans. Amer. Math. Soc. 121, 1-35.

[10] K. Zhu, Operator theory in function spaces, Monographs and Textbooks in Pure and Applied Mathematics 139 (Marcel Dekker, Inc., New York, 1990).

School of Mathematical Sciences

Peking University

Bejing 100871

China

e-mail: jxiao@sxx0.math.pku.edu.cn

\author{
Institute of Analysis \\ TU-Braunschweig \\ D-38106 Braunschweig \\ Germany \\ e-mail: xiao@badbit.math2.nat.tu-bs.de
}

